\title{
Germline VHL Mutation Discovered in Association with EGFR-Positive Lung Cancer and Metachronous Hepatocellular Carcinoma: A Case Report
}

\author{
Justin J. Kuhlman ${ }^{a}$ Quinn J. Frier ${ }^{b}$ Daniel Sumarriva ${ }^{c}$ Matthew Oberley ${ }^{d}$ \\ Danielle Bolton $^{e}$ Ruby A. Deveras ${ }^{f}$ \\ aDepartment of Internal Medicine, Mayo Clinic, Jacksonville, FL, USA; 'Department of \\ Internal Medicine, Prisma Health, Columbia, SC, USA; 'Department of Molecular Science, \\ Caris Life Sciences, Phoenix, AZ, USA; dDepartment of Hematopathology, Caris Life \\ Sciences, Phoenix, AZ, USA; eHereditary Risks and Genetics, Center for Oncology, Halifax \\ Health, Daytona Beach, FL, USA; ' Department of Medical Oncology and Hematology, Halifax \\ Health, Daytona Beach, FL, USA
}

\section{Keywords}

Von Hippel-Lindau · Lung adenocarcinoma · Hepatocellular carcinoma · Squamous cell carcinoma Osimertinib

\begin{abstract}
$\mathrm{VHL}$ is a tumor suppressor gene located on chromosome 3 that is classically associated with tumors of the eye and CNS, renal cell carcinoma, and pheochromocytoma. We describe what appears to be the first report of an association between a germline VHL mutation and nonsmall cell lung cancer and metachronous hepatocellular carcinoma (HCC). Our case involves a 63-year-old nonsmoking male who was initially diagnosed with EGFR mutation-positive metastatic nonsquamous, non-small cell lung adenocarcinoma, who subsequently developed HCC and squamous cell carcinoma of the femur despite first-line treatment with EGFRblocking osimertinib. Caris molecular profiling unexpectedly identified a shared underlying VHL mutation in all 3 lesions. Genetic mapping through a machine learning-based tool called Genomic Prevalence Score (GPSai ${ }^{\mathrm{TM}}$ ) helped determine that the femur tumor was a metastatic lesion as opposed to a separate primary and that the HCC was a distinct primary malignancy. We not only highlight the association between these tumors and a VHL mutation but also emphasize the value of next-generation sequencing and a molecular disease
\end{abstract}


classifier in a patient with multiple primaries, how it helps guide therapy, and its value in guiding future studies.

\section{Background}

Von Hippel-Lindau (VHL) is a tumor suppressor gene located on chromosome 3 that when mutated is classically associated with VHL syndrome. Predisposing one to the formation of benign and malignant tumors, VHL syndrome is an autosomal dominant condition that most commonly presents with hemangioblastomas of the eye and CNS, renal cell carcinomas, pancreatic neuroendocrine tumors, and pheochromocytomas [1]. Affecting approximately 1 in 36,000 births, VHL syndrome is an extremely rare condition that usually presents as early as the second decade of one's life with symptoms nearly always arising before 65 years of age [2]. Primarily derived as a hereditary condition, approximately $80 \%$ of cases are inherited from an affected parent while $20 \%$ seem to occur de novo without any preceding family history [1].

We report what appears to be the first case of an association between VHL mutation and non-small cell lung cancer (NSCLC) and metachronous hepatocellular carcinoma (HCC). Our patient developed what originally appeared to be 3 primary tumors, none of which are classically associated with VHL disease, including EGFR mutation-positive lung adenocarcinoma, EGFR wild-type HCC, and EGFR mutation-positive squamous cell carcinoma (SCC) of the sternum and femur. While none of these tumors are classically associated with VHL syndrome, the appearance of these 3 apparently independent lesions in a short time frame was suspicious for an underlying germline mutation, thereby leading to our discovery of a VHL mutation. We describe these tumors, their relationship to VHL, their molecular profiles, and how a molecular disease classifier helped determine the relationship of these histologically distinct tumors.

\section{Case Presentation}

A 63-year-old male without any significant family history presented with cough, 20-pound weight loss, and right lower lung infiltrate on chest X-ray. CT of the chest confirmed a spiculated mass in the right lower lobe, and biopsy revealed moderately differentiated adenocarcinoma as shown in Figure 1a. Curative surgery was attempted but resulted in a right lung pneumonectomy with positive margins and pleural metastases, resulting in final pathologic staging T4 N2 M1. Caris molecular profiling revealed an EGFR exon 19 deletion (pE746_T751delinsVA). The patient was initiated on systemic targeted therapy with osimertinib. Ten months later, surveillance imaging discovered a subtle hepatic mass in the right lobe of the liver. A needle-core biopsy revealed well-differentiated HCC. The patient had no evidence of disease from his original lung cancer while on osimertinib, so he was offered curative resection. Pathology confirmed a low-grade HCC as seen in Figure 1b.

Imaging with MRI 2 months following liver resection revealed a new ring-enhancing lesion in the sternum. CT-guided biopsy of the sternal lesion was consistent with a diagnosis of SCC as demonstrated in Figure 1c. The sample was insufficient for comprehensive tissuebased NGS testing, and Guardant360 cfDNA testing only identified a variant of unknown 


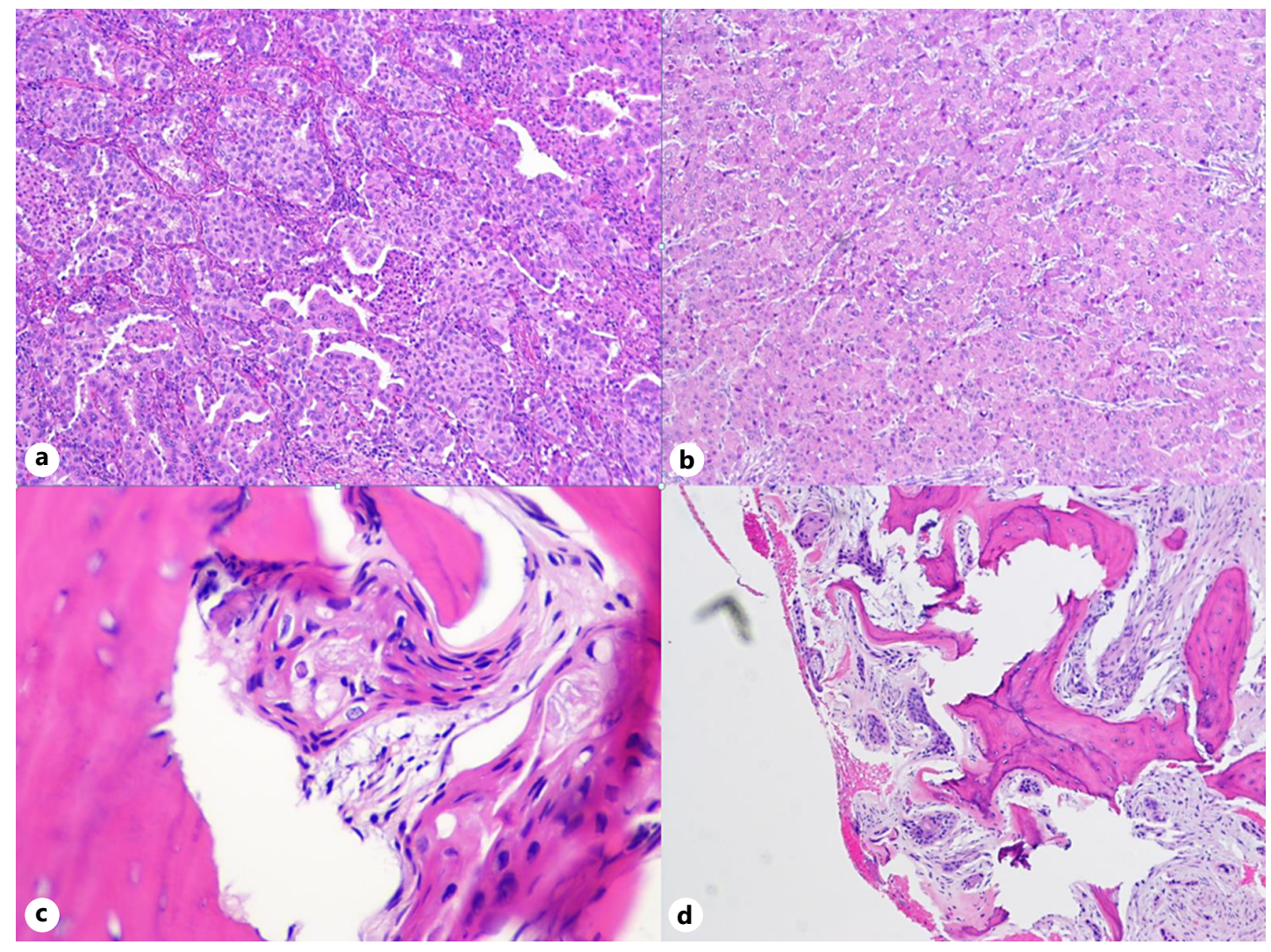

Fig. 1. Moderately differentiated adenocarcinoma of the right lung, EGFR positive (HE; original magnification, $\times 100)(\mathbf{A})$; low-grade hepatocellular carcinoma, EGFR negative (HE; original magnification, $\times 100)(\mathbf{B})$; squamous cell carcinoma of the sternum (HE; original magnification, $\times 400)(\mathbf{C})$; squamous cell carcinoma of the femur, EGFR positive (HE; original magnification, $\times 100)($ D). HE, hematoxylin and eosin.

significance (ATM [p.R2832H]). Osimertinib was continued. Only 10 days later, the patient presented with extreme pain in his right knee and thigh. MRI revealed a large destructive bone lesion involving the shaft of the right femur with severe cortical thinning as shown in Figures 2a and b, a slightly displaced pathological fracture of the anterior cortex. Biopsy of the lesion was consistent with SCC upon pathological review (shown in Fig. 1d). Available tissue was sent for comprehensive DNA and RNA next-generation sequencing (NGS) in order to assess for driver mutation(s) and help determine tissue of origin. Caris Molecular Intelligence assay of the femur lesion revealed an EGFR Exon 19 mutation (pE746_T751delinsVA) identical to the lung tumor. Histologic re-review of the lung adenocarcinoma revealed no evidence of squamous differentiation.

Given the potential molecular relatedness with divergent histology between the lung and femur metastasis, a molecular disease classifier known as Genomic Prevalence Score (GPSai ${ }^{\mathrm{TM}}$ ) was then employed. Utilizing several hundred DNA and RNA features to identify a signature for a tumor and after comparing the signature to specimens in the Caris database, the GPSai ${ }^{\mathrm{TM}}$ tool identifies all the tumors in the database with a matched signature and then lists them according to prevalence. The GPS prediction for the patient's lung adenocarcinoma gave a very high match score of $98 \%$, and the tumors in the database with a matched signature were labeled as lung adenocarcinoma. The GPS prediction for the patient's femur lesion also gave a high match score at $85 \%$, and the tumors in the database with a matched signature were labeled as SCC. Interestingly, $9 \%$ of the tumors 


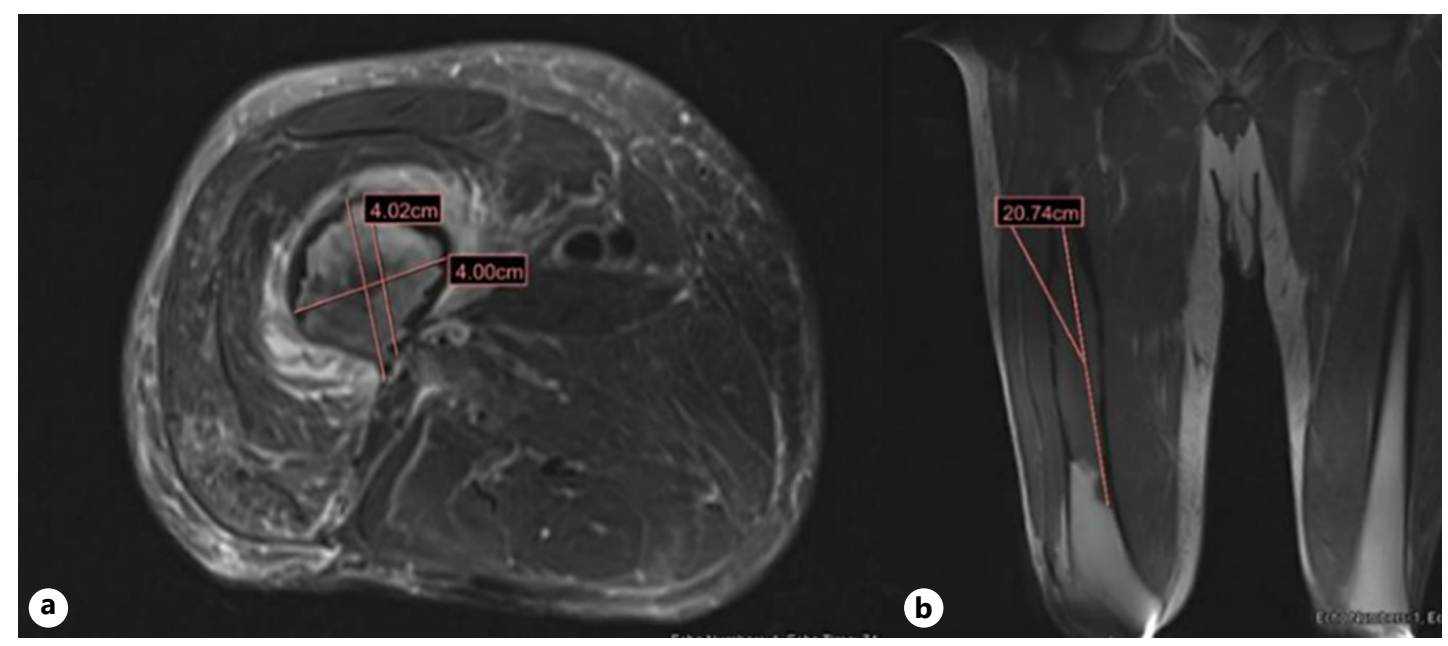

Fig. 2. A Cross-sectional view and B coronal view from MRI of the right femur demonstrating a large destructive bone lesion involving the femur shaft and measuring approximately $20.7 \mathrm{~cm}$ in length and $4 \mathrm{~cm}$ in diameter.

with a matched signature to the femur lesion carried the label of lung adenocarcinoma. The patient's liver tumor also gave a high match score at $94 \%$, and the matched signatures corresponded with HCC.

Molecular profiling also revealed an identical background VHL mutation in all 3 tumors, specifically p.L188V Exon 3 c.562C > G. The Guardant360 cfDNA was revisited for this VHL mutation. In correlation with Caris comprehensive molecular testing, a VHL L188V alteration was detected in the patient's blood sample at an allele fraction suspicious for germline origin. The patient was then referred for genetic counseling and testing. A Quest myVantage Hereditary Comprehensive Cancer Panel in fact confirmed the VHL variant, c.562C > G (p.Leu188Val), to be a heterozygous pathogenic germline mutation.

\section{Discussion}

This case describes the discovery of a background VHL mutation by Caris molecular profiling in 3 cancerous lesions. Initially diagnosed with an EGFR exon 19 deletion NSCLC, the patient then developed a HCC and SCC of the femur and sternum despite EGFR-targeting therapy with osimertinib. Although therapeutic approaches to EGFR-positive lung adenocarcinomas include TKIs (i.e., erlotinib and gefitinib), EGFR T790M mutation-targeting therapy (i.e., osimertinib) has demonstrated superior efficacy over earlier EGFR-TKIs as a first-line therapy with similar safety profiles [3]. For this reason, the patient was initially treated with osimertinib as the first-line therapy. With T790M mutations accounting for approximately $50 \%$ of cases of resistance to first- and second-generation EGFR-TKIs, osimertinib targets the T790M mutation and thereby eliminates this most common mechanism of resistance to earlier generation TKIs [4]. Tumors that harbor a driver mutation (i.e., EGFR), however, have been known to carry lower response rates to immunotherapy despite PD-L1 positivity or high tumor mutation burden. Nevertheless, our patient and his response to treatment was predicted by Caris NGS and the high tumor mutation burden due to his germline VHL mutation and underlying variant allele frequencies (VAFs). He responded well to combined chemoimmunotherapy and has continued immunotherapy alone as a maintenance therapy with stable disease at this time. 
Table 1. Next-generation sequencing results

\begin{tabular}{llll}
\hline Mutation & Femur VAF, \% & Lung VAF, \% & Liver VAF, \% \\
\hline VHL L188V & $\mathbf{4 7}$ & $\mathbf{5 0}$ & $\mathbf{5 1}$ \\
EGFR ex19del & 19 & 85 & - \\
SPECC I 680E & 85 & - & - \\
SOCS1 del & 40 & - & 61 \\
PTPN11 6409A & 57 & 64 & 27 \\
MSH6 1464T & 9 & 27 & - \\
MLLT3 & 15 & 19 & - \\
KIAA1549 S1877P & $\mathbf{5 5}$ & $\mathbf{5 0}$ & $\mathbf{5 0}$ \\
BCL11B R103C & $\mathbf{4 5}$ & $\mathbf{5 2}$ & $\mathbf{4 6}$ \\
AXIN1 P467t & $\mathbf{5 7}$ & $\mathbf{4 7}$ & $\mathbf{4 6}$ \\
ASXL1 1552V & $\mathbf{5 2}$ & $\mathbf{5 4}$ & $\mathbf{4 9}$ \\
CTNNB1 N387K & - & 15 & - \\
CAMTA1 V6271 & $\mathbf{5 6}$ & $\mathbf{4 8}$ & $\mathbf{4 6}$ \\
CREB3L1 G274L & - & - & 29
\end{tabular}

VAFs resulting at approximately 50\% indicate germline origin. Bold indicates positive germline benign variants or variants of uncertain significance shared by all 3 tumors.

VAF, variant allele frequency.

Histopathological examination initially seemed to indicate 3 separate primary tumors in our patient, with each tumor possessing multiple variants of uncertain significance according to NGS, some of which had a VAF suspicious for being a germline variant. Multiple variants were identified at such rates, including VHL L188V (shown in Table 1). These VAFs were all proven to be germline variants upon germline sequencing of the blood. The molecular disease classifier GPSai ${ }^{\mathrm{TM}}$ then discovered that the original NSCLC had molecular data that strongly predicted a diagnosis of lung adenocarcinoma while the metastatic SCC had molecular data that predicted a diagnosis of SCC with a secondary prediction of lung adenocarcinoma. The predicted lineage relatedness of the 2 sampled tumors by GPSai ${ }^{\mathrm{TM}}$, coupled with the same EGFR exon 19 deletion, strongly suggests that the femur tumor represents a metastasis of the original lung cancer, potentially from an area with squamous differentiation that was not sampled by the original biopsy. Alternatively, histologic transformation may have occurred. Although histological conversion of lung adenocarcinoma is typically toward small cell cancer [5], adenocarcinoma to SCC conversions with retained EGFR mutation have also been described [6]. Furthermore, histologic conversion is a welldescribed mechanism of resistance to osimertinib therapy [7]. In this context, the genetic signature of metastatic lesions can provide significant insight into the tumorigenesis of distinct lesions. Furthermore, such signatures assist in assessing response to treatment. For our patient, the EGFR VAF notably decreased from $85 \%$ in the lung to $19 \%$ in the femur lesion while on osimertinib therapy (shown in Table 1). Rather than representing a form of histologic transformation, the femur lesion more accurately depicts a naturally selected metastatic clone derived from the NSCLC as a result of both genetic and treatment pressure from TKI therapy.

The presence of a germline VHL mutation in the development of our patient's synchronous and metachronous tumors remains significant as each tumor represents an atypical presentation of VHL mutation. Located on chromosome 3, the VHL gene encodes the VHL protein 
(pVHL), which is responsible for the ubiquitination and degradation of hypoxia-inducible factor, a transcription factor that regulates genetic expression of angiogenic factors [8]. Decreased pVHL production thereby permits unregulated HIF activity, promoting angiogenesis central to tumor growth. Classically associated with vascular tumors of the eye and CNS, kidneys, and adrenal glands, VHL mutations have been documented in sporadic tumors and may be present in as many as $50 \%$ of sporadic hemangioblastomas and $50-60 \%$ of sporadic renal cell carcinomas $[9,10]$. In relation to NSCLC, EGFR gain and VHL loss have been associated with poor overall survival in patients with lung adenocarcinoma, suggesting their utilization simply as prognostic markers [11]. With the exception of SCC of the lung, NSCLC has not otherwise been associated with VHL genetic mutations [12]. A single report seems to detail an association between VHL disease and multiple metachronous lung adenocarcinomas [13]. Likewise, the association between VHL and HCC is not fully understood. One study demonstrated how low VHL expression in patients with HCC resulted in decreased survival and a poor clinical prognosis [14]. Our case appears to be the first to report an underlying genetic VHL mutation in the setting of NSCLC, HCC, and a metastatic lesion to the femur. More research is necessary in order to further investigate VHL's role in the tumorigenesis of these tumors.

\section{Conclusion}

Our report appears to be the first to highlight the relationship between these unusual metachronous tumors and a background VHL mutation in the context of EGFR-positive NSCLC. Although more research is indeed necessary to further elucidate VHL's role in the development of such tumors, our case suggests that molecular characterization of tumors provides information not obtainable by histological examination. Molecular signatures such as GPSai ${ }^{\mathrm{TM}}$ scoring contribute to a greater understanding of tumor genesis, as such tools have been found to have an accuracy of $94 \%$ in identifying tumor type when matching with a high probability [15]. Furthermore, commercially available NGS and GPS are tools available to clinicians and can provide significant utility in establishing diagnoses and assessing responses to treatments.

\section{Statement of Ethics}

Approval by an ethics committee was not obtained nor sought after due to the current manuscript representing a single-patient case report, describing what is known to be standard of care involving minimal risk. Written informed consent was obtained from the patient for the publication of this manuscript and any accompanying images.

\section{Conflict of Interest Statement}

The authors declare that there are no competing interests present.

\section{Funding Sources}

The authors received no financial support for the research, authorship, and/or publication of this article. 


\section{Author Contributions}

All authors contributed substantially to the conceptualization, drafting, and revisions of this manuscript.

\section{Data Availability Statement}

All data analyzed during this study are included in this article, but further enquiries or requests for additional data can be directed to the corresponding author.

\section{References}

1 Varshney N, Kebede AA, Owusu-Dapaah H, Lather J, Kaushik M, Bhullar JS. A review of von hippel-lindau syndrome. J Kidney Cancer VHL. 2017;4(3):20.

2 van Leeuwaarde RS, Ahmad S, Links TP, Giles RH. Von Hippel-Lindau syndrome. In: Adam MP, Ardinger HH, Pagon RA, Wallace SE, Bean LJH, Mirzaa G, et al., editors. Genereviews ${ }^{\circledR}$ [Internet]. Seattle (WA): University of Washington, Seattle; 1993-2021.

3 Soria J-C, Ohe Y, Vansteenkiste J, Reungwetwattana T, Chewaskulyong B, Lee KH, et al. Osimertinib in untreated EGFR-mutated advanced non-small-cell lung cancer. N Engl J Med. 2018;378(2):113-25.

4 Ricordel C, Friboulet L, Facchinetti F, Soria JC. Molecular mechanisms of acquired resistance to third-generation EGFR-TKIs in EGFR T790M-mutant lung cancer. Ann Oncol. 2018;29:i28-37.

5 Liu Y. Small cell lung cancer transformation from EGFR-mutated lung adenocarcinoma: a case report and literatures review. Cancer Biol Ther. 2018;19(6):445-9.

6 Izumi H, Yamasaki A, Ueda Y, Sumikawa T, Maeta H, Nakamoto S, et al. Squamous cell carcinoma transformation from EGFR-mutated lung adenocarcinoma: a case report and literature review. Clin Lung Cancer. 2018;19(1):e63-6.

7 Ren X, Cai X, Li J, Zhang X, Yu J, Song X, et al. Histological transformation of lung adenocarcinoma to small cell lung cancer with mutant C797S conferring acquired resistance to osimertinib. J Int Med Res. 2020;48(6): 300060520927918.

8 Zhou L, Yang H. The Von Hippel-Lindau tumor suppressor protein promotes c-Cbl-independent poly-ubiquitylation and degradation of the activated EGFR. PLoS One. 2011;6(9):e23936.

9 Kaelin WG. The Von Hippel-Lindau tumor suppressor gene and kidney cancer. Clin Cancer Res. 2004;10(18 Pt 2):6290S-5S.

10 Shankar GM, Taylor-Weiner A, Lelic N, Jones RT, Kim JC, Francis JM, et al. Sporadic hemangioblastomas are characterized by cryptic VHL inactivation. Acta Neuropathol Commun. 2014;2(1):167-12.

11 Zhao Y, Li Y, Wang S, Lu H, Chen J, Zhang Z, et al. The relationship between EGFR gain and VHL loss in lung adenocarcinoma and poor patient survival. Int J Clin Oncol. 2011;16(6):679-85.

12 Testa U, Castelli G, Pelosi E. Lung cancers: molecular characterization, clonal heterogeneity and evolution, and cancer stem cells. Cancers. 2018;10(8):248.

13 Ikeda K, Osumi H, Matsuishi K, Matsubara E, Fujino K, Shibata H, et al. Multiple lung adenocarcinomas associated with Von Hippel-Lindau disease. Ann Thorac Surg. 2014;98(4):1467-70.

14 Li G, Shen Y, Wang F, Hong S, Cai M. Correlation between Von Hippel-Lindau gene expression and tumor SUVmax and survival prognosis in hepatocellular carcinoma. Med Sci Monit. 2020;26:e920473.

15 Abraham J, Heimberger AB, Marshall J, Heath E, Drabick J, Helmstetter A, et al. Machine learning analysis using 77,044 genomic and transcriptomic profiles to accurately predict tumor type. Transl Oncol. 2021;14(3): 101016.

\section{Karger'k}

